\title{
APLICAÇÃO DA TÉCNICA DELPHI PARA VALIDAÇÃO DOS MÉTODOS A SEREM UTILIZADOS NO SISTEMA EM PLATAFORMA WEB PARA IMPLANTAÇÃO DE PLANO DE SEGURANÇA DA ÁGUA
}

\section{APPLICATION OF THE DELPHI TECHNIQUE FOR VALIDATION OF THE METHODS TO BE USED IN THE WEB PLATFORM SYSTEM FOR IMPLANTATION OF WATER SAFETY PLAN}

\section{Nolan Ribeiro Bezerra}

Doutoura em Engenharia Civil (Saneamento e Meio Ambiente) - UFV. Instituto Federal de Educação, Ciência e Tecnologia de Goiás. (nolanbezerra@gmail.com)

\section{Resumo}

Este trabalho tem como objetivo validar os métodos a serem empregados no sistema em plataforma Web, visando à implantação do Plano de Segurança da Água, face às diretrizes da Organização Mundial de Saúde e do Ministério da Saúde, por meio da utilização da técnica Delphi. Para tanto, foram selecionados os métodos mais utilizados nos países, tais como: Análise de Perigos e Pontos Críticos de Controle (APPCC) e a Matriz de Priorização do Risco. A execução da validação ocorreu por meio do auxílio de um questionário com quatro questões resumidas sobre os dois métodos, com espaço para comentários de opiniões dos especialistas. O resultado da validação dos métodos ocorreu em duas oficinas (Brasília-DF e Campinas-SP) de trabalho presencial, e as sugestões foram avaliadas, discutidas e incorporadas após consenso de todos os especialistas. A partir da validação, os especialistas concluíram por utilizar apenas a matriz de priorização de riscos com alteração, sugerindo que a árvore de decisão do sistema APPCC necessita de adequações. A matriz validada irá reduzir as incertezas e melhorar a eficiência da avaliação dos riscos.

Palavras-chave: Análise de perigos e pontos críticos de controle; matriz de priorização do risco; plano de segurança da água.

\section{Abstract}

This work aims to validate the methods to be used in the Web platform system, aiming at the implementation of the Water Safety Plan, in accordance with the guidelines of the World Health Organization and the Ministry of Health, through the use of the Delphi technique. In order to do so, the most used methods in the countries were selected, such as: Hazard Analysis and Critical Control Points (HACCP) and the Risk Prioritization Matrix. The validation was carried out through the aid of a questionnaire with four summary questions about the two methods with spaces for comments of the experts' opinions. The results of the validation of the methods occurred in two workshops (Brasília-DF and Campinas-SP) for face-to-face work and the suggestions were evaluated and discussed incorporated after consensus of all specialists. From the validation, the experts concluded by using only the risk prioritization matrix with change and suggesting that the decision tree of the HACCP system needs adjustments. The validated matrix will reduce uncertainties and improve the efficiency of risk assessment.

Keywords: Hazard analysis and critical control point; risk prioritization matrix; water safety plan.

\section{INTRODUÇÃO}

Em reconhecimento às limitações inerentes ao princípio amostral, técnico-analíticas ou financeiras e à dificuldade ou mesmo impossibilidade de monitoramento em tempo real, para assegurar a confiabilidade da água distribuída, novas ferramentas de gestão de riscos, para a efetiva garantia da segurança da água para consumo humano ao invés do simples monitoramento da água distribuída, vêm sendo propostas. Para suprir essas limitações, a Organização Mundial da Saúde (OMS), na terceira edição de 2004 (WHO, 2004) e mantida na quarta edição de 2011 de seu Guidelines for Drinking-Water Quality (WHO, 2011), recomenda 
aos prestadores de serviços públicos de saneamento básico, responsáveis pelo sistema de abastecimento de água, a implantação de Planos de Segurança da Água (PSA).

O PSA é um instrumento de identificação dos perigos e caracterização dos riscos em sistema e em solução alternativa coletiva de abastecimento de água para consumo humano, desde 0 manancial onde será realizada a captação da água até o consumidor, com a finalidade de estabelecer medidas de controle para eliminar ou reduzir os perigos e riscos para níveis aceitáveis (WHO, 2011). Segundo Vieira (2011), o Plano privilegia uma abordagem preventiva em detrimento da metodologia clássica de monitoramento de "fim-de-linha", para um processo de gestão de segurança, compreendendo todas as etapas que compõem o sistema de abastecimento, desde a fonte até o consumidor.

A OMS recomenda a implementação do PSA pautada em cinco atividades-chave, a saber: (i) metas de saúde baseadas em uma avaliação de riscos; (ii) avaliação da capacidade do sistema de abastecimento de água (da bacia hidrográfica contribuinte para a captação, tratamento e distribuição) em produzir água com qualidade, para o atendimento das metas de saúde; (iii) monitoramento dos sistemas de abastecimento nos pontos considerados chave para 0 tratamento; (iv) planos de gestão documentado, nos quais constem a avaliação do sistema, o monitoramento, rotinas operacionais, medidas corretivas em caso de anormalidades, previsão de ampliações e melhorias; e (v) programa de vigilância independente, que avalia os itens de (i) a (iv) de responsabilidade dos prestadores de serviços (WHO, 2004; 2005; 2010; 2011).

No Brasil, os fundamentos legais para o desenvolvimento do PSA estão regulamentados na Portaria de Consolidação n 5 , de 03 de outubro de 2017 que revoga a antiga Portaria $\mathrm{n}$ 은 2.914, de 12 de dezembro de 2011, do Ministério da Saúde. O art. 129 da Portaria no 5/2017 explicita a necessidade de o responsável pelo sistema ou pela solução alternativa de abastecimento de água para consumo humano manter avaliação sistemática do sistema, sob a perspectiva dos riscos à saúde, com base na qualidade da água distribuída, cabendo ao setor Saúde, ou seja, a Vigilância em Saúde, a avaliação dos Planos de Segurança da Água, conforme os princípios recomendados pela OMS ou definidos em diretrizes vigentes no país (BRASIL, 2017).

A diretriz recomendada pelo Ministério da Saúde tem como pressuposto as recomendações da OMS e o resultado do projeto piloto 'Plano de Segurança da Água', financiado pelo Ministério da Saúde e pelas agências norte-americanas US Center for Disease Control and Prevention (CDC) e US Environmental Protection Agency (USEPA), e desenvolvido pela Universidade Federal de Viçosa (UFV), sob a coordenação do Departamento de Engenharia Civil, em parceria com o Serviço Autônomo de Água e Esgotos de Viçosa (SAAE Viçosa-MG).

As publicações da OMS e do Ministério da Saúde fornecem as recomendações metodológicas, com a finalidade de orientar o prestador de serviço, responsável pelo sistema ou pela solução alternativa coletiva de abastecimento de água, na elaboração e implantação do Plano de Segurança da Água em todas as suas etapas, levando em consideração as informações sobre o uso e ocupação da bacia contribuinte ao manancial, o histórico das características das águas, as características físicas do sistema, as práticas operacionais e a qualidade da água (WHO, 2005; BARTRAM et al., 2009; BRASIL, 2013).

Do ponto de vista conceitual e metodológico, os principais elementos do PSA estão baseados nos princípios e conceitos de múltiplas barreiras e boas práticas (WHO, 2010). A implantação do PSA requer a identificação de eventos perigosos, os perigos e a caracterização dos riscos à saúde por meio da aplicação do método de caracterização dos riscos, tais como a matriz frequência $x$ consequências, a qual pode ser construída em base quantitativa, semiquantitativa ou qualitativa, e do método de Análise de Perigos e Pontos Críticos de Controle (APPCC), do inglês Hazard Analysis and CriticalControl Points (HACCP) (BARTRAM et al., 2009; AS/NZS, 2004; WHO, 2004; 2011; ABNT, 2009b).

Essas metodologias de caracterização de riscos são, entretanto, carregadas de subjetividades, ou seja, são dependentes de julgamentos empíricos por parte dos responsáveis pela aplicação do PSA. No entanto, elas podem ser ajustadas de acordo com a instituição e com os diversos tipos e tamanhos de sistemas de abastecimento de água para 
consumo humano. Por conseguinte, cabem aos municípios à adoção e adequação desses métodos, para implementação de PSA, considerando os aspectos econômicos, socioambientais e a realidade local.

Nesse contexto, é essencial que as adequações dessas metodologias utilizadas na identificação dos eventos perigosos e caracterização dos riscos, desde a bacia hidrográfica até a rede de distribuição, requeiram a consulta aos especialistas que pesquisam e trabalham diretamente com sistemas de abastecimento de água para as adequações e validação dos métodos a serem utilizados.

Segundo Davison (2007), a identificação dos perigos e a caracterização dos riscos devem ser realizadas por uma equipe técnica multidisciplinar com amplo conhecimento, permitindo, assim, que essas ferramentas possam ser articuladas e completadas por técnicas que utilizam o consenso de opinião de especialistas, como a metodologia Delphi.

Dessa forma, o presente artigo busca validar os métodos inerentes ao Plano de Segurança da Água, face às diretrizes da Organização Mundial de Saúde e do Ministério da Saúde, por meio da utilização da técnica Delphi. Os resultados dessa validação serão incorporados na proposta metodológica a ser inserida no desenvolvimento do Sistema de Informação para Implantação de Plano de Segurança da Água-SIPSA, com apoio financeiro do Instituto Federal de Educação, Ciência e Tecnologia - IFG, por meio do Edital no 006/2014-PROPPG, de 18 de março de 2014, e a Chamada Pública MEC/SETEC/CNPq № 17/2014, sob a coordenação da autora deste artigo.

\section{MÉTODOS UTILIZADOS NA IMPLANTAÇÃO DO PLANO DE SEGURANÇA DA ÁGUA}

Mundialmente, os métodos e as técnicas utilizados para identificação de perigos e caracterização dos riscos em sistemas de abastecimento de água, do manancial à distribuição de água, visando a estabelecer medidas de controle para eliminá-los ou reduzilos para níveis aceitáveis, estão baseados nos princípios e conceitos de múltiplas barreiras, na Análise de Perigos e Pontos Críticos de Controle (APPCC), na avaliação quantitativa de risco químico e microbiológico, avaliação e gerenciamento de risco, matriz de priorização do risco, análises estatísticas, análises multicritérios, gestão de qualidade e nas redes Bayesianas (HAVELAAR, 1994; HAAS et al., 1999; GUNNARSDÓTTIR et al., 2008; OLIVEIRA, 2010; ABNT, 2009b; BEZERRA, 2011).

Neste artigo, foram avaliados e propostos para validação os métodos que vêm sendo mais utilizados nos países, tais como: Análise de Perigos e Pontos Críticos de Controle (APPCC) e a matriz de priorização do risco.

\subsection{Análise de Perigos e Pontos Críticos de Controle (APPCC)}

A metodologia APPCC, originalmente utilizada na indústria química e, posteriormente, incorporada à indústria alimentícia, é uma ferramenta que possibilita a identificação de perigos associados às diversas etapas de produção, e a determinação de pontos críticos de controle e respectivos limites críticos operacionais por meio de protocolos de monitoramento, a fim de apontar medidas preventivas e, ou, corretivas que minimizem ou eliminem riscos à saúde pública (WHO, 1998).

Segundo WHO (1998), o sistema APPCC baseia-se em um sistema de engenharia conhecido como Análise de Modos de Falhas e Efeitos (Failure Mode and Effect Analysis FMEA), em que se observam, em cada etapa do processo, as falhas que podem ocorrer, suas causas prováveis e seus efeitos, para então estabelecer mecanismos de controle.

O APPCC é um sistema que identifica, avalia e controla perigos que são significativos para a segurança de alimentos (CODEX, 1993). Foi concebido em doze componentes, sendo cinco etapas preliminares desenvolvidas pela Comissão do Codex Alimentarius e sete princípios (WHO, 1998), conforme descrito na Figura 1.

A APPCC determina os pontos críticos de controle (PCC) nas etapas do processo, nas quais os perigos podem ser controlados ou eliminados, estabelece limites críticos necessários para controlar os perigos, monitora os limites críticos para cada PCC a intervalos definidos e estabelece ações corretivas se o processo estiver fora dos limites estabelecidos em normas. A determinação dos PCC é realizada por meio do uso da árvore de decisão, que representa uma sequência de questões. 
Figura 1- Etapas e princípios do APPCC

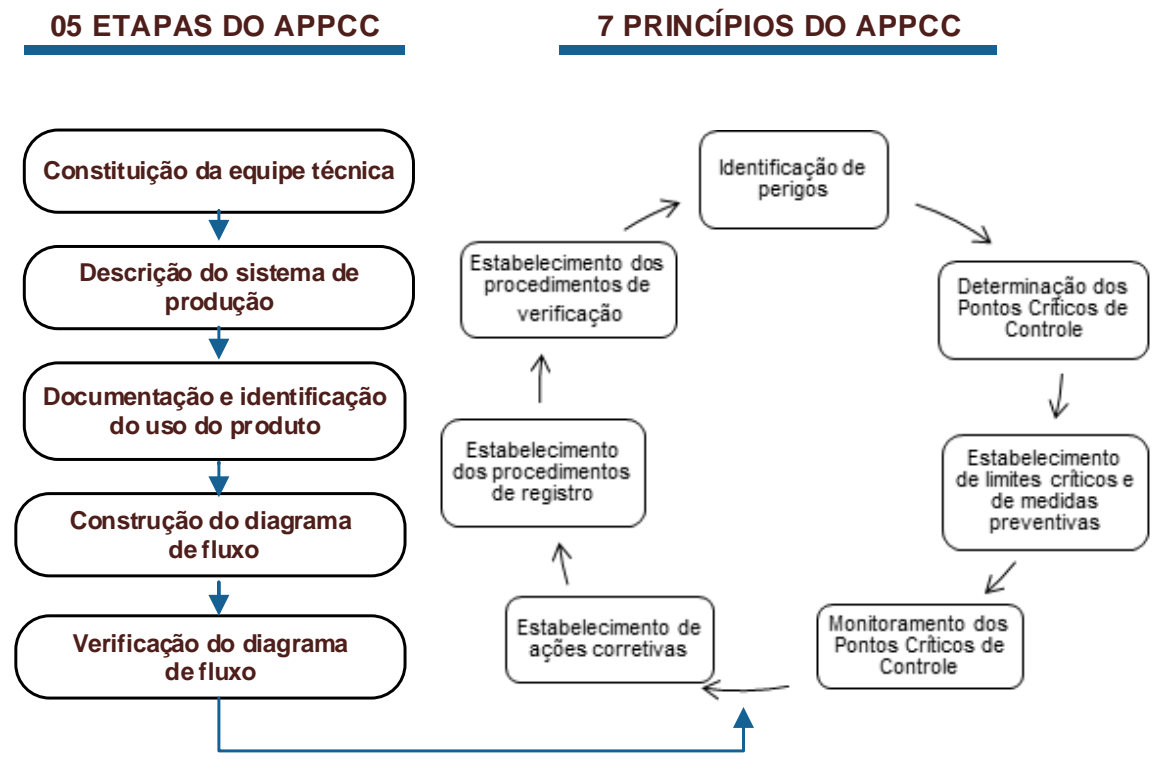

Fonte: Adaptado de WHO (1998).

O controle é a medida necessária para assegurar e manter o cumprimento dos critérios estabelecidos no sistema APPCC, e pontos de controle são pontos, procedimentos, operações ou etapas nas quais o controle pode ser exercido ou aplicado. Pontos Críticos são caracterizados por situações ou locais nos quais os perigos identificados na etapa anterior podem estar presentes. Por sua vez, Pontos Críticos de Controle (PCC) se referem aos pontos do processo ou operação que devem ser supervisionados para possibilitar eliminar ou reduzir os perigos (OLIVEIRA, 2010).

Com as devidas adaptações, o sistema APPPC vem ganhando atenção e aplicação na produção de água para consumo humano e sua concepção serviu de base para a proposição das etapas preconizadas no Plano de Segurança da Água (HAVELAAR, 1994; GUNNARSDÓTTIR et al., 2008; VIDAL, 2012).

\subsection{Matriz de priorização do risco}

A matriz de priorização do risco é originada das normas Standards Australia e Standards New Zealand (AS/NZS, 2004), as quais foram atualizadas pela ISO 31000:2009 (ABNT, 2009b). No Brasil, a norma geral de Gestão de Riscos, que fornece uma estrutura genérica para estabelecer os contextos e para a identificação, análise, avaliação, tratamento, monitoramento e comunicação de riscos nas organizações, foi regulamentada pelas normas ISO/IEC 31000:2009 (ABNT, 2009b), Guia ISO 73:2009 (ABNT, 2009a) e NBR ISO/IEC 31010:2012 (ABNT, 2012), as quais tratam das técnicas para o processo de avaliação de riscos e fornecem orientações sobre a seleção e aplicação de técnicas sistemáticas para 0 processo de avaliação do risco.

O método consiste na determinação da probabilidade de um perigo/evento perigoso ocorrer e a magnitude de suas consequências à saúde. A ferramenta muito utilizada é a matriz frequência $x$ severidade, a qual pode ser construída em base qualitativa, semiquantitativa ou quantitativa. O processo de gestão de riscos é definido pela ISO/IEC 31000:2009 como sendo:

Aplicação sistemática de políticas, procedimentos e práticas de gestão para as atividades de comunicação, consulta, estabelecimento do contexto, e na identificação, análise, avaliação, tratamento, monitoramento e análise crítica dos riscos (ABNT, 2009b, p.7).

A análise qualitativa requer utilização de termos que bem expressem a probabilidade de ocorrência e a severidade das consequências de um determinado risco. Já na análise semiquantitativa, são atribuídos valores numéricos aos descritores de probabilidade e de consequência, de forma que de seu cruzamento 
resulte produto numérico; pode ser determinada por meio da matriz descrita na Figura 2. O cruzamento dos valores de probabilidade frequência (certo, quase certo, frequente) e consequência resulta em um risco (elevado, alto, médio e baixo). As escalas podem ser lineares ou logarítmicas, ou podem ter alguma outra relação; as fórmulas utilizadas também podem variar.

Figura 2 - Exemplo de matriz de priorização de risco por meio da técnica semiquantitativa

\begin{tabular}{|c|c|c|c|c|c|}
\hline \multirow[b]{2}{*}{ Frequência } & \multicolumn{5}{|c|}{ Severidade } \\
\hline & $\begin{array}{l}\text { Efeito nulo } \\
\text { ou } \\
\text { insignifi- } \\
\text { cante } \\
\text { Classificaç } \\
\text { ão: } 1\end{array}$ & $\begin{array}{l}\text { Efeito de } \\
\text { cumpri- } \\
\text { mento leve } \\
\text { Classifica- } \\
\text { ção: } \\
2\end{array}$ & $\begin{array}{c}\text { Efeito } \\
\text { organoléti-co } \\
\text { moderado } \\
\text { Classifica- } \\
\text { ção: } \\
3\end{array}$ & $\begin{array}{l}\text { Efeito } \\
\text { regulamentá } \\
\text { rio grave } \\
\text { Classificaçã } \\
\text { o: } 4\end{array}$ & $\begin{array}{l}\text { Efeito } \\
\text { Catastrófi-co } \\
\text { na saú-de } \\
\text { pública } \\
\text { Classifica- } \\
\text { ção: } \\
5\end{array}$ \\
\hline $\begin{array}{l}\text { Quase Certo/uma vez } \\
\text { ao dia - Classificação: } \\
5\end{array}$ & 5 & 10 & 15 & 20 & 25 \\
\hline $\begin{array}{l}\text { Provável/uma vez por } \\
\text { semana Classificação: } \\
4\end{array}$ & 4 & 8 & 12 & 16 & 20 \\
\hline $\begin{array}{l}\text { Moderada/uma vez ao } \\
\text { mês Classificação: } 3\end{array}$ & 3 & 6 & 9 & 12 & 15 \\
\hline $\begin{array}{l}\text { Improvável/uma vez ao } \\
\text { ano Classificação: } 2\end{array}$ & 2 & 4 & 6 & 8 & 10 \\
\hline $\begin{array}{l}\text { Excepcional/uma vez a } \\
\text { cada } 5 \text { anos } \\
\text { Classificação: } 1\end{array}$ & 1 & 2 & 3 & 4 & 5 \\
\hline $\begin{array}{l}\text { Pontuação do risco } \\
>15 \\
\text { Classificação do risco } \\
\text { alto }\end{array}$ & & ixo & $\begin{array}{r}\text { 6-9 } \\
\text { Médio }\end{array}$ & & Ito \\
\hline
\end{tabular}

Fonte: Bartram et al. (2009).

$\mathrm{Na}$ análise quantitativa, a probabilidade e as consequências são combinadas com o resultando em determinado nível de risco, por meio, quando possível, de análise e cálculos estatísticos (por exemplo, com a aplicação de Avaliação Quantitativa de Risco Microbiológico - AQRM) (ABNT, 2009b).

Observa-se que a avaliação do nível de risco pode ser interpretada como risco muito alto, alto, médio e baixo, conforme matriz proposta nas diretrizes da OMS por Bartram et al. (2009). A construção da matriz é definida pelo usuário de acordo com o objetivo do estudo (ISO/IEC 31000:2009) (ABNT, 2009b). O risco é calculado pelo produto da ocorrência em relação à sua severidade. Um mesmo produto numérico pode ser classificado em níveis distintos de risco, dependendo do que for assumido como de maior ou menor peso.
Portanto, nessa abordagem, assim como na matriz qualitativa, o usuário, ao definir esses descritores, pode determinar que uma severidade catastrófica (peso 5), em função da frequência excepcional (peso 1), gere um risco baixo com nível 5, conforme o exemplo da matriz sugerida pela OMS. Essa falha, ao definir a matriz para aplicação em sistema de abastecimento de água, pode gerar um problema grave, uma vez que um evento catastrófico, mesmo ocorrendo com uma frequência de a cada cinco anos ou mais, pode ter um efeito letal para a população.

Em função do elevado grau de subjetividade, é preciso definir e validar, a partir da consulta de opinião de especialistas e técnicos que trabalham com sistema de abastecimento de água, os descritores por meio da atribuição de pesos aplicáveis aos objetivos de saúde pública, para fins de fornecimento de água potável e que não ofereça risco à saúde. 


\section{METODOLOGIA}

A metodologia foi concebida em duas etapas, conforme apresentado na Figura 3. A primeira etapa consta da seleção e adequação dos métodos inerentes ao Plano de Segurança da Água (PSA), e a segunda etapa apresenta os passos para a validação dos métodos por meio da técnica Delphi.

3.1. Primeira Etapa: Seleção e adequação dos métodos inerentes ao Plano de Segurança da Água (PSA)

Para o desenvolvimento deste estudo, inicialmente, foi realizado uma revisão bibliográfica no âmbito internacional e nacional, para seleção dos métodos mais utilizados na implantação do PSA nos países.

A seleção e adequação dos métodos levaram em consideração a metodologia proposta pela OMS nos Guidelines for Drinking-Water Quality, que trata da temática desde 2004 até os dias atuais (WHO, 2004; 2005; 2011), a norma ISO 31000 de Gestão de Risco (ABNT 2009b), bem como as diretrizes do Ministério da Saúde (BRASIL, 2011) e o resultado do Projeto Piloto Brasil já referido neste artigo.

Após seleção dos métodos, o passo seguinte foi a esquematização e adequação da metodologia, considerando a concepção tecnológica dos sistemas de abastecimento de água, conforme realidade brasileira.

Figura 3 - Etapas da metodologia adotada

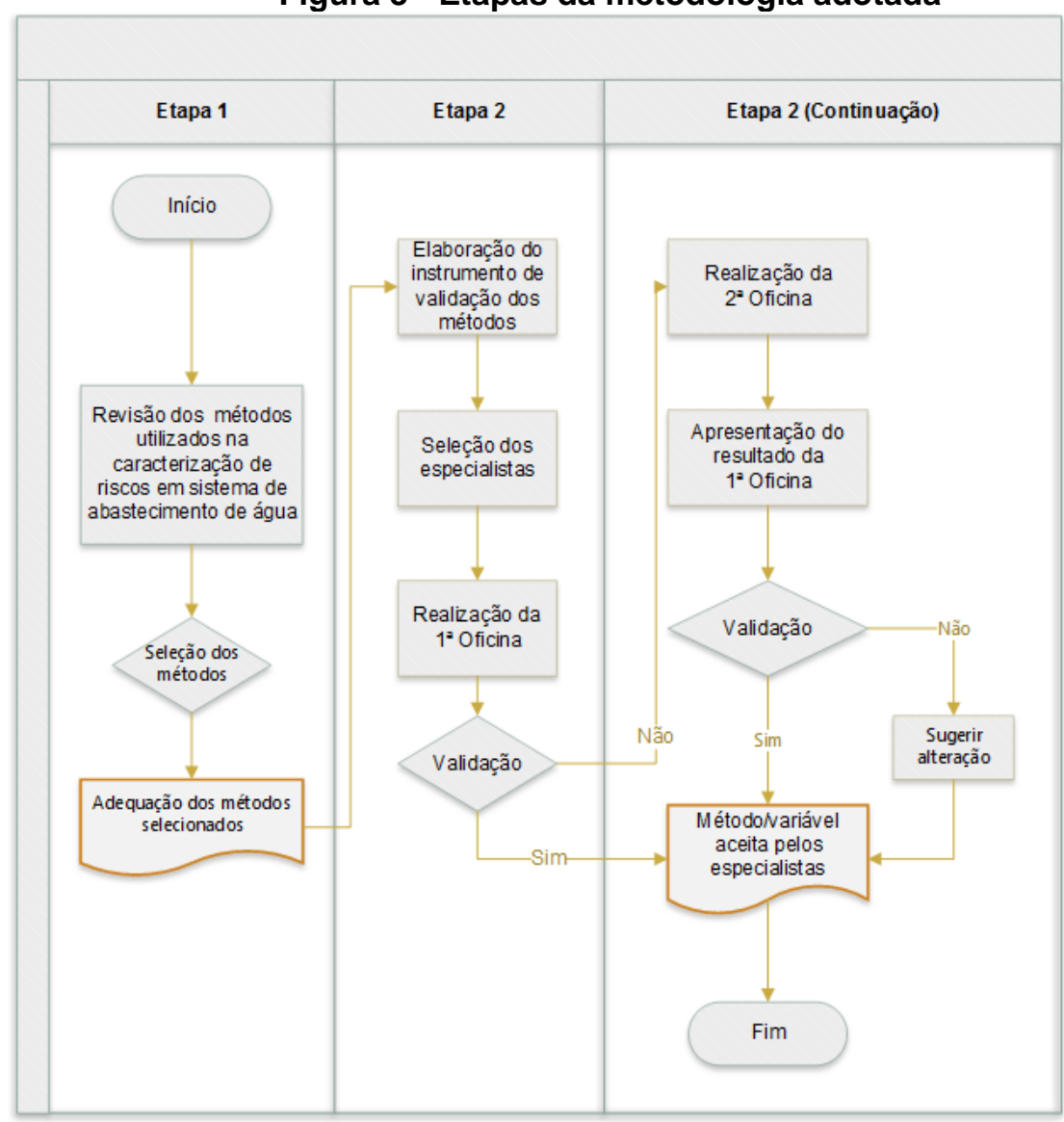

Fonte: A Autora.

3.1. Segunda Etapa: Validação dos métodos a serem adotados para implantação do Plano de Segurança da Água (PSA)

A validação da metodologia foi obtida, por meio de consulta aos especialistas, utilizando a técnica DELPHI que, segundo Piola et al. (2002), pode ser vista como uma forma de estruturar um processo de comunicação, de maneira a possibilitar que um conjunto de especialistas gere uma opinião coletiva qualificada sobre problemas complexos. Para Wright (1986), a técnica Delphi 
envolve a definição do objeto a ser validado, por meio da elaboração de um instrumento de consulta, intercalado com informações resumidas e espaços para comentários de opiniões provenientes de respostas anteriores dos especialistas consultados, bem como a seleção dos especialistas, análise das respostas da primeira rodada e segunda rodada e validação final do consenso dos especialistas.

A seleção dos especialistas levou em consideração o fato destes atuarem nas áreas de recursos hídricos, microbiologia, tratamento de água, epidemiologia, substâncias químicas, vigilância da qualidade da água e avaliação de riscos à saúde.

A técnica Delphi pode ser realizada por meio de consulta a distância ou presencial. A execução dessa validação ocorreu por meio de duas oficinas de trabalho presencial, e as sugestões foram avaliadas e discutidas para posterior incorporação das mesmas aos métodos selecionados.

A validação dos métodos selecionados ocorreu a partir da elaboração do instrumento com informações resumidas dos dois métodos, tendo espaço para comentários dos especialistas. A primeira oficina foi realizada no período de dois dias na cidade de Brasília-DF. O resultado dessa oficina foi consolidado e elaborado outro questionário com as respostas obtidas (consenso e não consenso) para realização de uma segunda rodada. A segunda oficina de fechamento ocorreu em Campinas-SP, onde foi possível fazer a validação dos métodos objeto deste estudo.

\section{RESULTADOS E DISCUSSÃO}

Após avaliação do referencial teórico e a experiência da autora deste artigo na implantação do projeto piloto realizado no Serviço Autônomo de Água e Esgotos de Viçosa (SAAE Viçosa-MG), bem como no Plano de Segurança da Água (PSA) no Saneamento de Goiás S.A. (SANEAGO), em desenvolvimento, foi possível propor a utilização da Matriz de Priorização de Riscos em conjunto com a Árvore de Decisão, prevista no método de Avaliação de Pontos Críticos de Controle (APPCC), métodos mais utilizados na implantação do PSA.

Para implantação do PSA, sugeriu-se a utilização da Matriz de Priorização de Riscos em conjunto com a aplicação da Árvore de Decisão, para fins de identificação de Pontos Críticos de Controle (PCC), Ponto de Controle (PC), ou Pontos de Atenção (PC), para os riscos classificados como moderado a alto (6 a 40). A indicação do uso dos métodos, em conjunto, levou em consideração a experiência portuguesa (VIEIRA et al., 2006). A matriz tem a finalidade de priorizar os riscos de baixo a alto e, quando for detectado risco moderado acima de seis pontos, será obrigatório o uso da Arvore de Decisão para identificar se aquele perigo é um PCC, PC ou PA.

Segundo Mortmore e Wallace (2001), a Árvore de Decisão consiste em uma série de perguntas que devem ser respondidas para verificar como cada perigo encontrado pode ser controlado/removido em cada etapa do sistema de abastecimento de água. Cabe destacar que Vieira e Moraes (2005) buscaram adaptar a terminologia, não alterando os princípios da árvore de decisão original do Codex Alimentarius, em experiência adquirida na elaboração e aplicação de um Plano de Segurança da Água na empresa Águas do Cávado S.A.

A matriz de risco selecionada para validação foi a matriz semiquantitativa, proposta por Bartram et al. (2009), apresentada na Figura 2, em conjunto com a aplicação do sistema APPCC. Dessa forma, para a determinação dos Pontos Críticos de Controle (PCCs), adotou-se o método da árvore de decisão descrita e modificada por Oliveira (2010) na Figura 4.

Os métodos propostos nas Figuras 2 e 4 foram apresentados na oficina com o propósito de utilização em conjunto, conforme já explicitado, seguindo depois com a discussão e obtenção da opinião dos 14 (quatorze) especialistas participantes da primeira oficina, por meio de um questionário estruturado com 4 (quatro) questões com espaços para comentários e opiniões dos mesmos.

As questões foram estruturadas para avaliação das variáveis da descrição da frequência, severidade, nível de risco e pertinência ou não do uso em conjunto da árvore de decisão, conforme descrito no Quadro 1. Os critérios avaliados pelos especialistas para cada questão foram: "Aceitar indicação", "Aceitar indicação com alteração" e "Rejeitar indicação". Ao marcar "Aceitar indicação com alteração" e "Rejeitar indicação", o especialista deveria sugerir alteração complementar. 
A questão 04 do Quadro 1 teve como objetivo avaliar a pertinência da utilização da Árvore de Decisão para a identificação de PCC a partir dos riscos priorizados como moderado, quando da utilização da Matriz de Priorização de Riscos. No instrumento, cada indicação poderia receber apenas um voto, dentre as opções apresentadas como "Sim", "Não" e "Não se Aplica". O especialista, ao não considerar a indicação da Árvore de Decisão, deveria votar com "Não" e, se julgasse inapropriado o uso dos dois métodos, deveria votar "Não se Aplica".
Os resultados das opiniões dos especialistas de consenso e não consenso, para as quatro questões (Quadro 1) validadas na primeira oficina realizada no período de dois dias na cidade de Brasília-DF, não apresentaram consenso para duas questões, conforme proposta inicial. Quando analisada a questão que trata da avaliação da descrição da "Frequência" e do "Significado", foram rejeitados os descritores da "Frequência", adotados os descritores do "Significado" e mantido apenas a da Frequência, conforme resultado apresentado no Quadro 2.

Quadro 1 - Variáveis dos métodos avaliados pelos especialistas nas oficinas nas cidades de BrasíliaDF e Campinas-SP, 2016

\begin{tabular}{|c|c|c|}
\hline Questões/ Variáveis & Descritores & Significado dos descritores \\
\hline \multirow{5}{*}{$\begin{array}{l}\text { Questão 1: Avaliação da } \\
\text { descrição da frequência }\end{array}$} & Quase Certo & Diária a semanal \\
\hline & Muito Provável & Quinzenal a mensal \\
\hline & Provável & Semestral a anual \\
\hline & Pouco Provável & Acima de um ano até 5 anos \\
\hline & Raro & Acima de 5 anos \\
\hline \multirow{5}{*}{$\begin{array}{l}\text { Questão 2: Avaliação da } \\
\text { descrição da severidade }\end{array}$} & Insignificante & Sem impacto detectável \\
\hline & Baixa & $\begin{array}{l}\text { Pequeno impacto sobre a qualidade estética / organoléptica da água } \\
\text { e, ou, baixo risco à saúde que pode ser minimizado em etapa } \\
\text { seguinte do sistema de abastecimento }\end{array}$ \\
\hline & Moderada & $\begin{array}{l}\text { Elevado impacto estético e, ou, com potencial risco à saúde que } \\
\text { pode ser minimizado em etapa seguinte do sistema de } \\
\text { abastecimento. }\end{array}$ \\
\hline & Elevada & $\begin{array}{l}\text { Potencial impacto à saúde que não pode ser minimizado em etapa } \\
\text { seguinte do sistema de abastecimento, necessitando de realização } \\
\text { de monitoramento operacional e medidas de controle. }\end{array}$ \\
\hline & Catastrófica & Risco elevado à saúde com interrupção do fornecimento de água. \\
\hline \multirow{4}{*}{$\begin{array}{l}\text { Questão 3: Avaliação da } \\
\text { descrição da análise de } \\
\text { risco }\end{array}$} & $\begin{array}{l}\text { Risco Baixo } \\
\leq 5\end{array}$ & $\begin{array}{l}\text { Risco baixo, tolerável, sendo controlável por meio de procedimentos } \\
\text { de rotina. }\end{array}$ \\
\hline & $\begin{array}{l}\text { Risco Moderado } \\
6 \text { a } 12\end{array}$ & $\begin{array}{l}\text { Risco moderado, necessidade de atenção e de identificação de } \\
\text { pontos críticos de controle (PCC), pontos críticos (PC) ou pontos de } \\
\text { atenção (PA). }\end{array}$ \\
\hline & $\begin{array}{l}\text { Risco Alto } \\
16 \text { a } 40\end{array}$ & $\begin{array}{l}\text { Risco alto é não tolerável, necessidade de adoção de medidas de } \\
\text { controle e, ou, ações de gestão ou de intervenção física a médio e } \\
\text { longo prazo, sendo necessária a identificação de pontos críticos de } \\
\text { controle (PCC), pontos críticos (PC) ou pontos de atenção (PA), } \\
\text { estabelecimento de limites críticos e monitoramento dos perigos } \\
\text { para cada ponto identificado. }\end{array}$ \\
\hline & Risco Extremo & $\begin{array}{l}\text { Risco não tolerável, necessidade de adoção imediata de plano de } \\
\text { emergência. }\end{array}$ \\
\hline $\begin{array}{l}\text { Questão 4: Avaliação da } \\
\text { descrição da Árvore de } \\
\text { Decisão adaptada }\end{array}$ & \multicolumn{2}{|c|}{$\begin{array}{l}\text { A Árvore de Decisão para identificação de PCC com adaptações para sistemas de } \\
\text { abastecimento de água é adequada? }\end{array}$} \\
\hline
\end{tabular}
Fonte: A Autora. 


\section{Figura 4 - Árvore de decisão - Identificação de PCC com adaptações para} sistemas de abastecimento de água

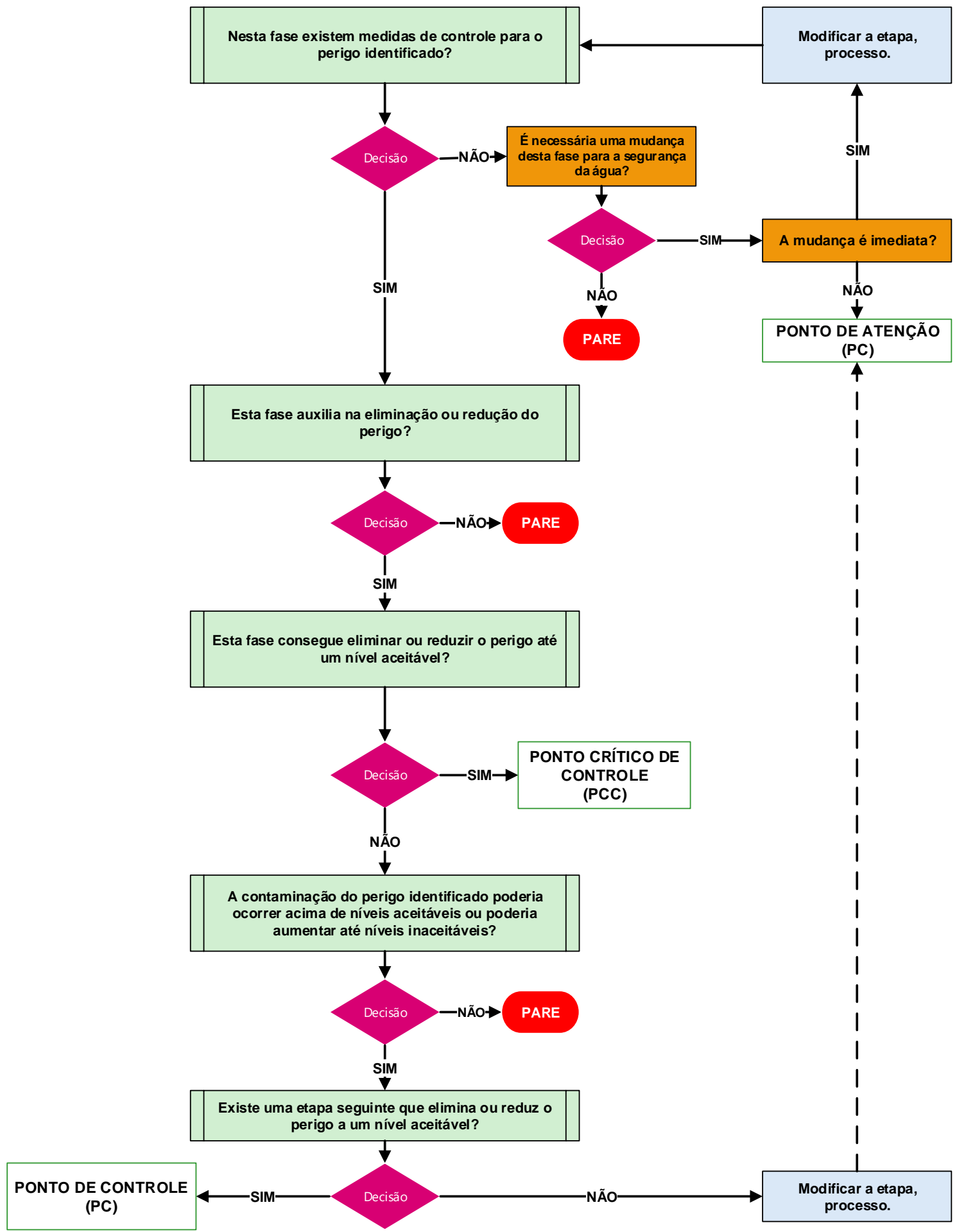

Fonte: Oliveira (2010).

As questões sobre a avaliação da descrição da "severidade" e a avaliação da descrição da "análise de risco" (Questões 2 e 3) não obtiveram consenso nas proposições e foram reavaliadas em uma segunda rodada. Já na questão 4 , houve consenso quanto à aplicação da Árvore de Decisão poder ser utilizada, desde que realizado teste.

Segundo Oliveira (2010), a identificação dos pontos críticos de controle com base na aplicação 
da árvore de decisão apresenta necessidade de maiores adequações para ser aplicada em sistemas de tratamento de água. No entanto, ambas as metodologias buscam avaliar os perigos acima do limite crítico e propor medidas de controle.

Quadro 2 - Resultado da opinião dos especialistas para questão 01 validada na 1 Oficina em 18 e 19 de fevereiro de 2016

\begin{tabular}{|llll|}
\hline \multicolumn{1}{|c}{ FREQUÊNCIA } & \multicolumn{1}{c|}{ Proposta Inicial } & \multicolumn{2}{c|}{ Opinião Validada na 1o Oficina } \\
\hline Quase Certo & Diária a semanal & FREQUÊNCIA & SIGNIFICADO \\
\hline Muito Provável & Quinzenal a mensal & Quinzenal a mensal & Rejeitado indicação \\
\hline Provável & Semestral a anual & Semestral a anual & Rejeitado indicação \\
\hline Pouco Provável & Acima de um ano até 5 anos & Acima de um ano até 5 anos & Rejeitado indicação \\
\hline Raro & Acima de 5 anos & Acima de 5 anos & Rejeitado indicação \\
& & & \\
\hline
\end{tabular}

Fonte: A Autora.

A segunda oficina de fechamento foi realizada em Campinas-SP, onde foi possível fazer a validação dos métodos objeto deste estudo. A Figura 5 apresenta o resultado final da Matriz de Priorização de Risco aprovada e validada. $\mathrm{Na}$ matriz validada, foram atribuídos valores numéricos aos descritores de frequência (5 a 1) e severidade (1 a 16), de forma que, de seu cruzamento, resulte produto numérico do nível de risco.

Para suprir a falha da matriz desenvolvida por Bartram et al. (2009), a matriz proposta na Figura 5 considerou que todos os perigos/eventos perigosos com severidade catastrófica, mesmo que o evento seja raro (acima de 5 anos), serão considerados como risco extremo, necessitando de adoção imediata de um plano de emergência.

Dominguez-Chicas (2010) adaptou a matriz de priorização de risco para aplicação em estação de tratamento de água (escala piloto), que faz captação de efluentes tratados da estação de tratamento de esgoto no Reino Unido. O autor considerou as falhas identificadas na matriz proposta por Bartram et al. (2009), no entanto, não levou em conta a distinção entre o grau de severidade de elevado e catastrófico dos 94 perigos (42\%) correspondentes aos grupos de pesticidas, orgânicos e microbiológicos.

Em estudo de caso realizado por Carvalho et al. (2015), na estação de tratamento de água de responsabilidade da Companhia de Saneamento Básico do Estado de São Paulo (Sabesp), foi levado em consideração o perigo crítico, como o impacto na saúde pública, e que pode gerar grandes danos ambientais excedendo os limites legais de forma sistêmica. No entanto, os autores não consideram a severidade dos perigos, como risco elevado à saúde, necessitando interromper o fornecimento de água.

Cabe destacar que a experiência de implantação de PSA no Brasil segue as recomendações propostas na diretriz da OMS e do Ministério da Saúde, sendo necessária uma atenção especial ao aplicar a matriz proposta nestas referências, uma vez que alguns estudos apresentam fragilidade e erros na determinação dos riscos.

\section{CONCLUSÃO}

O método aqui validado revela-se como poderosa ferramenta para a avaliação da efetividade da identificação dos perigos e para a caracterização dos riscos em sistemas de abastecimento de água, que desde a bacia hidrográfica até a distribuição constituem etapas fundamentais, pois delas dependem a priorização dos perigos a serem controlados e as medidas de controle a serem especificadas. Mundialmente, a matriz de priorização de riscos prevista no PSA é a mais utilizada, face às diretrizes da Organização Mundial de Saúde e do Ministério da Saúde.

A caracterização dos riscos é usualmente realizada por meio da matriz de priorização de risco, e sua aplicação envolve incertezas. Uma forma de lidar com incertezas é realizar a validação dos descritores para refinar estimativas de probabilidades associadas às demais variáveis de interesse. Dessa forma, acredita-se que os objetivos delineados para este artigo tenham sido cumpridos, uma vez que a matriz de priorização de risco foi validada pelos especialistas como o método mais indicado para a implementação de 
planos de segurança da água em sistemas de abastecimento de água.

Essa ferramenta permite a identificação e a priorização de perigos, a caracterização de riscos e o estabelecimento de medidas de controle. No entanto, cabe recomendar outros métodos eficientes, como, por exemplo, a aplicação da abordagem probabilística da rede Bayesiana, técnicas de análise multicritério e de geoprocessamento, as quais vêm se mostrando como ferramentas interdisciplinares para avaliar os níveis de impactos dos eventos perigosos na qualidade da água, bem como simular cenários de perigos futuros.

Figura 5 - Matriz Semiquantitativa de Priorização de Risco

\begin{tabular}{|c|c|c|c|c|c|c|}
\hline \multirow{3}{*}{\multicolumn{2}{|c|}{ Frequência }} & \multicolumn{5}{|c|}{ Severidade } \\
\hline & & \multirow{2}{*}{$\frac{1}{\text { Muito Baixa }}$} & \multirow{2}{*}{$\begin{array}{c}2 \\
\text { Baixa }\end{array}$} & \multirow{2}{*}{$\frac{4}{\text { Moderada }}$} & \multirow{2}{*}{$\begin{array}{c}8 \\
\text { Elevada }\end{array}$} & \multirow{2}{*}{$\begin{array}{c}16 \\
\text { Crítica }\end{array}$} \\
\hline & & & & & & \\
\hline 5 & $\begin{array}{c}\text { Diária a } \\
\text { semanalmente }\end{array}$ & $\begin{array}{l}\text { Baixo } \\
(5)\end{array}$ & $\begin{array}{l}\text { Moderado } \\
\text { (10) }\end{array}$ & $\begin{array}{l}\text { Alto } \\
(20)\end{array}$ & $\begin{array}{l}\text { Alto } \\
(40)\end{array}$ & $\begin{array}{c}\text { Extremo } \\
\text { Plano de } \\
\text { Emergência }\end{array}$ \\
\hline 4 & Quinzenal a mensal & $\begin{array}{l}\text { Baixo } \\
(4)\end{array}$ & $\begin{array}{l}\text { Moderado } \\
\text { (8) }\end{array}$ & $\begin{array}{l}\text { Alto } \\
(16)\end{array}$ & $\begin{array}{l}\text { Alto } \\
(32)\end{array}$ & $\begin{array}{c}\text { Extremo } \\
\text { Plano de } \\
\text { Emergência }\end{array}$ \\
\hline 3 & Semestral a anual & $\begin{array}{l}\text { Baixo } \\
(3)\end{array}$ & $\begin{array}{l}\text { Moderado } \\
\text { (6) }\end{array}$ & $\begin{array}{l}\text { Moderado } \\
\text { (12) }\end{array}$ & $\begin{array}{l}\text { Alto } \\
(24)\end{array}$ & $\begin{array}{c}\text { Extremo } \\
\text { Plano de } \\
\text { Emergência }\end{array}$ \\
\hline 2 & $\begin{array}{c}\text { Acima de um ano ate } 5 \\
\text { anos }\end{array}$ & $\begin{array}{l}\text { Baixo } \\
(2)\end{array}$ & $\begin{array}{l}\text { Baixo } \\
(4)\end{array}$ & $\begin{array}{l}\text { Moderado } \\
\text { (8) }\end{array}$ & $\begin{array}{l}\text { Alto } \\
(16)\end{array}$ & $\begin{array}{c}\text { Extremo } \\
\text { Plano de } \\
\text { Emergência }\end{array}$ \\
\hline 1 & Acima de 5 anos & $\begin{array}{l}\text { Baixo } \\
(1)\end{array}$ & $\begin{array}{l}\text { Baixo } \\
(2)\end{array}$ & $\begin{array}{l}\text { Baixo } \\
(4)\end{array}$ & $\begin{array}{l}\text { Moderado } \\
\text { (8) }\end{array}$ & $\begin{array}{c}\text { Extremo } \\
\text { Plano de } \\
\text { Emergência }\end{array}$ \\
\hline
\end{tabular}

Legenda:

\begin{tabular}{ccl}
\hline Nível & Descritor & \multicolumn{1}{l}{ Significado da Severidade } \\
\hline 1 & Muito Baixa & Sem impacto detectável. \\
\hline 2 & Baixa & $\begin{array}{l}\text { Impacto sobre a qualidade estética ou organoléptica da água, sem causar rejeição da água, } \\
\text { podendo ser mitigado em etapa seguinte do sistema de abastecimento de água. }\end{array}$ \\
\hline 3 & Moderada & $\begin{array}{l}\text { Impactos com risco moderado à saúde, abaixo do padrão de potabilidade, podendo ser } \\
\text { mitigado em etapa(s) seguinte(s) do sistema de abastecimento de água. }\end{array}$ \\
\hline 4 & Elevada & $\begin{array}{l}\text { Impactos com risco elevado à saúde, acima do padrão de potabilidade, que não podem ser } \\
\text { mitigado em etapa(s) seguinte(s) do sistema de abastecimento. }\end{array}$ \\
\hline 5 & Crítica & $\begin{array}{l}\text { Impactos com risco extremo à saúde, acima do padrão de potabilidade, com interrupção do } \\
\text { fornecimento de água e necessidade de execução de plano de contingência. }\end{array}$ \\
\hline
\end{tabular}

Análise do Risco:

Risco Baixo $\leq 5$ : Risco baixo

Risco Moderado 6 a 12: Risco tolerável

Risco Alto 16 a 40: Risco não tolerável

Risco Extremo: Risco não tolerável, necessidade de adoção imediata de plano de emergência.

Fonte: A Autora.

\section{REFERÊNCIAS}

ABNT - ASSOCIAÇÃO BRASILEIRA DE NORMAS TÉCNICAS. ABNT/ISO Guia 73, Gestão de riscos Vocabulário. Rio de Janeiro, ABNT, 2009a.

ABNT/NBR/ISO:31000, Gestão de riscos -

Princípios e diretrizes. 1. ed. Rio de Janeiro: ABNT, 2009b.
ABNT/NBR/ISO;IEC:31010, Gestão de riscos - Técnicas para o processo de avaliação de riscos. 1. ed. Rio de Janeiro: ABNT, 2012. 96p.

AS/NZS - AUSTRALIA, NEW ZEALAND. AS/NZS 4360:2004 Risk Management Standard. 3. ed. Standard Australia and Standard New Zealand, 2004. 
BARTRAM, J. Water Safety Plan manual: step-by-step risk management for drinking-water suppliers. Geneve: World Health Organization, 2009.

BEZERRA. N. R. Aplicação de redes Bayesianas na identificação de perigos em sistemas de abastecimento de água para consume humano: Estudo de caso no município de Viçosa - Minas Gerais. 2011. 183p. Tese (Doutorado em Engenharia Civil) Universidade Federal de Viçosa, Viçosa, 2011.

BRASIL. Portaria de Consolidação n 05, de 03 de outubro de 2017. Estabelece os procedimentos e responsabilidades relativos ao controle e vigilância da qualidade da água para consumo humano e seu padrão de potabilidade. Ministério da Saúde, Brasília, série E, 2017. Publicação № 190 - DOU de 03/10/17 Seção 1 - Suplemento. p.360.

- Ministério da Saúde. Secretaria de Vigilância em Saúde. Departamento de Vigilância em Saúde Ambiental e Saúde do Trabalhador. Plano de segurança da água: garantindo a qualidade e promovendo a saúde: um olhar do SUS. Ministério da Saúde, Secretaria de Vigilância em Saúde, Departamento de Vigilância em Saúde Ambiental e Saúde do Trabalhador. - Brasília: Ministério da Saúde, 2013. 60 p.

CARVALHO. R. C. M.; JACOB. R. R.; DURÂES. E. A. S. Implantação do Plano de Segurança da Água na Sabesp. Estudo de caso - ETA de Vargem. In: CONGRESSO INTERNACIONAL DE SEGURANÇA DA ÁGUA, 2015, Brasília. Anais eletrônicos. Brasília: MS, 2015.

CODEX ALIMENTARIUS COMMISSION. Guidelines for the application of the Hazard Analysis Critical Control Point (HACCP) system. Rome: Food and Agriculture Organization/ World Health Organization, 1993.

DAVISON, A. Water Safety Plan workbook for drinkingwater. Singapore: World Health Organization Western Pacific Regional Office, 2007.

DOMINGUEZ-CHICAS, A.; SCRIMSHAW, M. D. Hazard and risk assessment for indirect potable reuse schemes: an approach for use in developing Water Safety Plans. Water Research, v. 44, n. 20, p. 61156123, 2010.

GUNNARSDÓTTIR, M. J.; GISSURARSON, L. R. HACCP and Water Safety Plans in Icelandic water supply: preliminary evaluation of experience. Journal of Water and Health, v. 6, n. 3, p. 377-382, 2008.

HAAS, C. N.; ROSE, J. B.; GERBA, C. P. Quantitative microbial risk assessment. New York: John Wiley \& Sons, 1999.
HAVELAAR, A. H. Application of HACCP to drinking water supply. Food Control, v. 5, n. 3, p. 145-152, 1994.

MORTIMORE, S.; WALLACE, C. APPCC enfoque práctico. 2. ed. Zaragosa, Espana: Edictorial Acribia S.A., 2001. 427p.

OLIVEIRA, D. C. Aplicação da "análise de perigos e pontos críticos de controle" no tratamento de água para consumo humano. 2010. 132f. Dissertação (Mestrado em Engenharia Civil) - Universidade Federal de Viçosa, Viçosa, 2010.

PIOLA, S. F.; VIANNA, S. M.; VIVAS-CONSUELO, D. Estudo Delphi: atores sociais e tendências do sistema de saúde brasileiro. Caderno de Saúde Pública, Rio de Janeiro, n.18, p.181-190, 2002.

VIDAL, A. P.; MARROQUÍN, C. P. A.; LOZADA, P. T. Identificación y prorización de peligros como herramientas de la gestión del riesgo en sistemas de distribución del agua potable. Ingeniería y Universidad, v. 16, n. 2, p. 449-470, 2012.

VIEIRA, J. M.; MORAIS, C.; VALENTE, J. C. T.; PEIXOTO, F. Experiência da aplicação do plano de segurança da água na Águas do Cávado, S.A. Braga: Universidade do Minho, Portugal, 2006.

A strategic approach for Water Safety Plans implementation in Portugal. Journal of Water and Health, Portugal, v. 9, n. 1, p. 107-116, 2011.

; MORAIS, C. Manual para a elaboração de planos de segurança da água para consumo humano. Lisboa: Instituto Regulador de Águas e Resíduos, Universidade do Minho; 2005. 175p. (Série GuiasTécnicos n.ำ7).

WORLD HEALTH ORGANIZATION. Guidelines for Drinking-Water Quality. 4. ed. WHO Chronicle, v. 38, p. 104-108, 2011.

Water Safety Plans - Managing drinkingwater quality from catchment to consumer. Geneve, 2005. 244p.

Water Safety Plans. Geneve, 2010.

Guidance on the Regulatory Assessment of HACCP. Geneve, 1998.

Guidelines for Drinking-Water Quality. World Health Organization, 2004.

WRIGHT, J. T. C. A técnica Delphi: uma ferramenta útil para o planejamento do Brasil? In: ENCONTRO BRASILEIRO DE PLANEJAMENTO EMPRESARIAL "COMO PLANEJAR 86", 3., 1985, São Paulo. Anais.... São Paulo: SPE -Sociedade Brasileira de Planejamento Empresarial, 1986. p.199-207. 